\title{
A Group-Theoretical Approach to Black-Hole Radiation (*).
}

\author{
B. P. Dolan
}

Department of Mathematical Physics, St. Patrick's College - Maynooth, Ireland School of Theoretical Physics, Dublin Institute for Advanced Studies 10 Burlington Road Dublin 4, Ireland

(ricevuto il 23 Settembre 1988)

\begin{abstract}
Summary. - It is shown that, for static space-times, the phenomenon of Hawking radiation can be understood in terms of representations of the twodimensional Poincaré group. Thero zero-energy basis vector of one representation contains positive-energy basis vectors of another representation. An action of the two-dimensional Poincaré group is globally defined over the whole of space-time, but only generates isometries at the event horizon and in the asymptotically flat region (where it degenerates to simple translations). Some simple space-times are discussed.
\end{abstract}

PACS 03.70 - Theory of quantized field.

\section{Introduction.}

The purpose of this paper is to draw attention to the role of the twodimensional Poincaré group in the phenomenon of Hawking radiation in static space-times (e.g., Schwarzschild and de Sitter space-times).

In the first section, the quantum field theory of a scalar field in a curved space-time is reviewed in order to establish notation. The necessary existence of timelike Killing vectors, for the definition of energy, is emphasized. As an illustration the formalism is applied to two inertial observers in Minkowski space.

In sect. 2 a class of static metries (including Schwarzschild and de Sitter) is considered, and the two-dimensional Poincaré group is shown to act on these space-times in a definite fashion, intimately connected with the existence of

(*) To speed up publication, the author of this paper has agreed to not receive the proofs for correction. 
Kruskal coordinates. Hawking radiation can be understood in terms of unitary transformations between representations of the two-dimensional Poincaré group.

Section 3 applies the formalism to some metrics which have not previously been considered in the literature. In particular, it is shown that a static homogeneous electromagnetic field will display the Hawking radiation phenomenon.

Finally, a summary is given in sect. 4 .

\section{1. - General formalism.}

It would seem $(1,2)$ that the concept of "particle number" in a given quantum state is an observer-dependent quantity, i.e. the vacuum is observer dependent. We shall first review this phenomenon, by way of setting up notation.

Consider a massless, classical, scalar field, $\phi$, subject to the wave equation in a space-time $\mathscr{K}$, with a metric $g$ (assumed to be globally hyperbolic and time orientable, so that the Cauchy problem is well posed, $\left.\left({ }^{3}\right)\right)$ :

$$
\square \phi=0 \text {. }
$$

A freely falling observer, $S$, might decompose $\phi$ into a complete set of orthonormal functions, $u_{k}(x)$ :

$$
\phi(x)=\sum_{k}\left\{a_{k} u_{k}(x)+a_{k}^{\ddagger} u_{k}^{*}(x)\right\},
$$

where each $u_{k}$ satisfies $\square u_{k}=0$. (If $k$ takes continuous values, the sum must be replaced by an integral.)

However, a different geodesic observer, $S^{\prime}$, might decompose $\phi$ into a different set of orthonormal functions, $u_{k^{\prime}}^{\prime}(x)$ :

$$
\phi(x)=\sum_{k^{\prime}}\left\{a_{k^{\prime}}^{\prime} u_{k^{\prime}}^{\prime}(x)+a_{k^{\prime}}^{\prime \prime} u_{k^{\prime}}^{\prime *}(x)\right\},
$$

where each $u_{k^{\prime}}^{\prime}$ satisfies $\square u_{k^{\prime}}^{\prime}=0 ; u_{k}$ would be obtained by $S$ by setting up a locally inertial coordinate system $(t, \boldsymbol{x})$, where $t$ is his proper time and is tangent to his world-line. Similarly $S^{\prime}$ would use $\left(t^{\prime}, \boldsymbol{x}^{\prime}\right)$ as locally inertial coordinates with $t^{\prime}$ being his proper time.

(1) N. O. BIRRELl and P. C. W. Davies: Quantum Fields in Curved Space (Cambridge University Press, 1982).

(') G. W. Gibbons and S. W. Hawking: Phys. Rev. D, 15, 2738 (1977).

( $\left.{ }^{3}\right)$ S. W. HAWKING and G. F. R. Ellis: The Large Structure of Space-Time (Cambridge University Press, 1973). 
Then $S$ would wish to choose $u_{k}$ so that

$$
\frac{\partial u_{k}(x)}{\partial t}=-i \omega(k) u_{k}
$$$$
\omega(k) \geqslant 0 \text {. }
$$

In general, $\omega(k)$ can only be made independent of $t$ if $\partial / \partial t$ is a Killing vector for the metric $g$ (at least locally, along a segment of the world-line of $S$, if not globally). If the metric and observer's world line, $\gamma$, are such that separation of variables can be used (as will be the case for metrics and observers considered in the sequel), then $u_{k}$ can be chosen so that

$$
u_{k}(x)=\frac{1}{2 \sqrt{\pi \omega(k)}} \exp [-i \omega(k) t] f_{k}(\boldsymbol{x}),
$$

where $f_{k}(\boldsymbol{x})$ is independent of $t$. Similar comments also apply to $S^{\prime}$. Then the Fourier coefficients can be obtained by Fourier transforming along the observers world-line, $\gamma\left(\boldsymbol{x}=\boldsymbol{x}_{0}=\right.$ const $)$ :

$$
\begin{aligned}
& a_{k}=\frac{1}{f_{k}\left(\boldsymbol{x}_{0}\right)} \sqrt{\frac{\omega(k)}{\pi}} \int_{\gamma} \exp [i \omega(k) t] \phi\left(t, \boldsymbol{x}_{0}\right) \mathrm{d} t, \\
& a_{k}^{\dagger}=\frac{1}{f_{k}^{*}\left(\boldsymbol{x}_{0}\right)} \sqrt{\frac{\omega(k)}{\pi}} \int_{\gamma} \exp [-i \omega(k) t] \phi\left(t, \boldsymbol{x}_{0}\right) \mathrm{d} t,
\end{aligned}
$$

and the Fourier coefficients of each observer can be related:

$$
a_{k}=\sum_{k^{\prime}}\left(\alpha_{k k^{\prime}} a_{k^{\prime}}^{\prime}+\beta_{k k^{\prime}}^{*} a_{k^{\prime}}^{\prime \dagger}\right)
$$

where

$$
\begin{aligned}
& \alpha_{k k^{\prime}}=\frac{1}{f_{k}\left(\boldsymbol{x}_{0}\right)} \sqrt{\frac{\omega(k)}{\pi} \int_{r} \exp [i \omega(k) t] u_{k^{\prime}}^{\prime}\left(t, \boldsymbol{x}_{0}\right) \mathrm{d} t,} \\
& \beta_{k k^{\prime}}=\frac{1}{f_{k}^{*}\left(\boldsymbol{x}_{0}\right)} \sqrt{\frac{\omega(k)}{\pi}} \int_{r} \exp [-i \omega(k) t] u_{k^{\prime}}^{\prime}\left(t, \boldsymbol{x}_{0}\right) \mathrm{d} t
\end{aligned}
$$

are Bogoliubov coefficients $\left({ }^{4}\right)$.

(4) N. N. Bogoliubov: Ž. Ėksp. Teor. Fiz., 34, 58 (1958) (Sov. Phys. JETP, 7, 51 (1958)). 
When we pass to quantum field theory, $\phi$ becomes an operator, $\hat{\phi}$, on the Hilbert space of quantum states, and the Fourier coefficients $a_{k}^{\dagger}, a_{k}$ become creation and annihilation operators $\hat{a}_{k}^{\dagger}, \hat{a}_{k}$. Note that the operators of $S$ act on the same Hilbert space as those of $S^{\prime}$. In particular, the vacuum according to $S$ is a state, $|0\rangle$, defined by

$$
\hat{a}_{k}|0\rangle=0
$$

while the vacuum according to $S^{\prime}$ is a state $\left|0^{\prime}\right\rangle$ defined by

$$
\hat{a}_{k^{\prime}}^{\prime}\left|0^{\prime}\right\rangle=0
$$

If $\beta_{k k^{\prime}} \neq 0$, then $S^{\prime}$ sees $|0\rangle$ as containing particles:

$$
\left\langle 0\left|\hat{N}_{k^{\prime}}^{\prime}\right| 0\right\rangle=\sum_{k}\left|\beta_{k k^{\prime}}\right|^{2}
$$

where $\hat{N}_{k^{\prime}}^{\prime}=\hat{a}_{k^{\prime}}^{\prime \dagger} \hat{a}_{k^{\prime}}^{\prime}$ is the number operator of $S^{\prime}$. At the same time $S$ sees $\left|0^{\prime}\right\rangle$ as containing particles

$$
\left\langle 0^{\prime}\left|\hat{N}_{k}\right| 0^{\prime}\right\rangle=\sum_{k^{\prime}}\left|\beta_{k k^{\prime}}\right|^{2}
$$

where $\hat{N}_{k}=\hat{a}_{k}^{\dagger} \hat{a}_{k}$ is the number operator of $S$. More generally, a state $\left|n_{k}\right\rangle$ which $S$ sees as having $n_{k}$ particles of momentum $k$ will appear to $S^{\prime}$ to have $n_{k^{\prime}}^{\prime}$ particles of momentum $k^{\prime}$, where

$$
n_{k^{\prime}}^{\prime}=\sum_{l}\left\{\left|\beta_{l k^{\prime}}\right|^{2}\right\}+n_{k}\left(1+2\left|\beta_{k k^{\prime}}\right|^{2}\right)
$$

and vice versa.

Thus, if $\beta_{k k^{\prime}} \neq 0$, then the vacuum of one observer contains particles of the other, and the energies will also differ. If the two vectors $\partial / \partial t$ and $\partial / \partial t^{\prime}$ were to commute, then they would have the same eigenfunctions and eigenvalues. Thus a necessary condition for $\beta_{k k^{\prime}} \neq 0$ is that

$$
\left[\frac{\partial}{\partial t}, \frac{\partial}{\partial t^{\prime}}\right] \neq 0
$$

This, however, does not seem to be sufficient, since it is only a local statement, whereas particle production requires a knowledge of the global structure of the space-time and the world-lines (in particular, the existence of an event horizon is an important ingredient, though examples are constructed in $\left(^{5}\right)$ where this is not essential).

$\left.{ }^{5}\right)$ N. SANChez: Phys. Lett. B, 87, 212 (1979). 
When $\beta_{k k^{\prime}}$ is nonzero and $\partial / \partial t$ and $\partial / \partial t^{\prime}$ are (at least locally) Killing, we have the following group-theoretical interpretation of particle production. $u_{k}$ (respectively $u_{k^{\prime}}^{\prime}$ ) are a basis of states of a representation of the (local) group of isometries. In this representation $\partial / \partial t$ (respectively $\partial / \partial t^{\prime}$ ) is diagonal. The isometry group is being represented on the space of functions. The Fourier transform is a unitary transformation between the two different representations. The representations must be different if $\partial / \partial t$ and $\partial / \partial t^{\prime}$ do not commute. When $\beta_{k k^{\prime}} \neq 0$, the positive eigenvalue basis states of one representation contain negative eigenvalue basis states of the other, and vice versa.

As a trivial example, consider two inertial observers, $S$ and $S^{\prime}$, in twodimensional Minkouski space,

$$
t^{\prime}=\gamma(t-v x), \quad x^{\prime}=\gamma(x-v t)
$$

where $\gamma=\left(1-v^{2}\right)^{-1 / 2}$ is a constant. Then

$$
\frac{\partial}{\partial t}=\gamma \frac{\partial}{\partial t^{\prime}}-v \gamma \frac{\partial}{\partial x^{\prime}}
$$

Hence $\partial / \partial t$ and $\partial / \partial t^{\prime}$ commute and have the same eigenfunctions. In particular, a right moving mode has

$$
u_{k}=\frac{1}{2 \sqrt{\pi \omega}} \exp [-i \omega(t-x)], \quad u_{k^{\prime}}^{\prime}=\frac{1}{2 \sqrt{\pi \omega^{\prime}}} \exp \left[-i \omega^{\prime}\left(t^{\prime}-x^{\prime}\right)\right],
$$

where $\omega=|k|$ for massless particles. Along the world-line of $S, x=0$ and $t^{\prime}=\gamma t$, $x^{\prime}=-\gamma v t$ thus

$$
\alpha_{\omega \omega^{\prime}}=\frac{1}{2 \pi} \sqrt{\frac{\omega}{\omega^{\prime}}} \int_{-\infty}^{\infty} \exp [i \omega t] \exp \left[-\gamma(1+v) \omega^{\prime} t\right] \mathrm{d} t \propto \partial^{\prime}\left(\omega-\sqrt{\frac{1+v}{1-v}} \omega^{\prime}\right)
$$

and $\beta_{\omega \omega^{\prime}}=-i \alpha_{-\omega, \omega^{\prime}}=0$. This is just the relativistic Doppler effect.

\section{2. - The two-dimensional Poincaré group.}

Now consider metries of the form

$$
\mathrm{d} s^{2}=-V(r) \mathrm{d} t^{2}+V^{-1}(r) \mathrm{d} r^{2}+r^{2}\left(\mathrm{~d} \theta^{2}+\sin ^{2} \theta \mathrm{d} \varphi^{2}\right),
$$

$V(r)=(1-2 m / r)$ is the Schwarschild metric, while $V(r)=\left(1+(\Lambda / 3) r^{2}\right)$ is the de 
Sitter metric. More generally $V(r)=\left(1-2 m / r+e^{2} / r^{2}-(\Lambda / 3) r^{2}\right)$ is the ReissnerNordstrom-de Sitter metric.

For all of these metrics $\partial / \partial t$ is a timelike Killing vector in the region $V>0$, but it is only tangent to geodesics in the region $V>0$ when $V \rightarrow 1\left(r=r_{0}\right)$. When $V=0\left(r=r_{E}\right)$ there is an event horizon, and the Schwarzschild coordinates are singular. Nonsingular coordinates at $r=r_{\mathrm{E}}$ can be found $(2,6)$, and are obtained by defining

$$
r^{*}=\int^{r} \frac{\mathrm{d} r}{V(r)}
$$

and

$$
v=t+r^{*}, \quad w=t-r^{*} .
$$

Then Kruskal coordinates are defined by

$$
v^{\prime}=\exp [v / \lambda], \quad w^{\prime}=-\exp [-w / \lambda]
$$

where $\lambda$ is a constant. (The sign of $r *$ is chosen for the Schwarzschild case. For a uniform treatment of de Sitter we would use the opposite sign, but the final result is the same.) The metric can be analytically continued to the regions with $v^{\prime}<0$ and $w^{\prime}>0$ :

$$
\begin{array}{cc}
v^{\prime}=\exp [v / \lambda], & w^{\prime}=\exp [-w / \lambda], \\
v^{\prime}=-\exp [v / \lambda], & w^{\prime}=\exp [-w / \lambda], \\
v^{\prime}=-\exp [v / \lambda], & w^{\prime}=-\exp [-w / \lambda] .
\end{array}
$$

For the Schwarzschild metric, an observer at $r_{0}=\infty$ is in region I, while for the de Sitter metric an observer at $r_{0}=0$ is in region $\mathrm{I}$.

The Penrose diagrams $\left({ }^{3}\right)$ for these two cases are shown in fig. 1.

With $t^{\prime}=\frac{1}{2}\left(v^{\prime}+w^{\prime}\right), r^{\prime}=\frac{1}{2}\left(v^{\prime}+w^{\prime}\right)$, the metric takes the form

$$
\mathrm{d} s^{2}=\lambda^{2} V(r) \exp \left[-2 r^{*} / \lambda\right]\left(-\mathrm{d} t^{2}+\mathrm{d} r^{\prime 2}\right)+r^{2}\left(\mathrm{~d} \theta^{2}+\sin ^{2} \theta \mathrm{d} \varphi^{2}\right) .
$$

Let $C(r)=\lambda V^{1 / 2} \exp \left[-r^{*} / 2\right]$. Then for these coordinates to be nonsingular at $r=r_{\mathrm{E}}$ we require $c\left(r_{\mathrm{E}}\right) \neq 0$ and is finite and $(\mathrm{d} c / \mathrm{d} r) \mid r_{\mathrm{E}}$ is finite. These conditions

(6) M. D. KruSKaL: Phys. Rev., 119, 1743 (1960). 

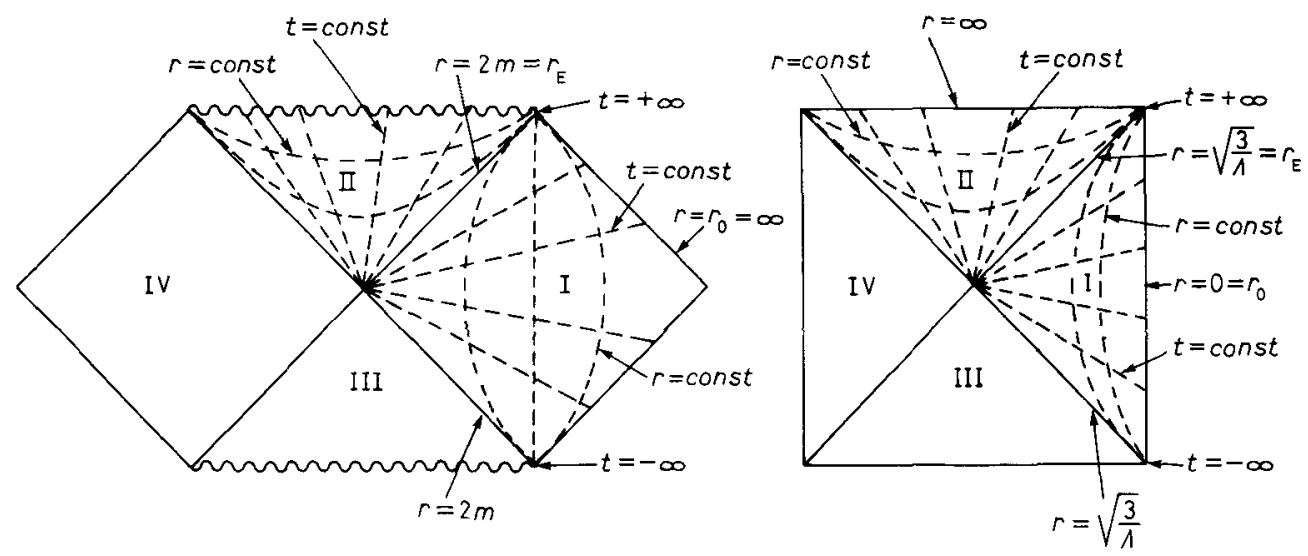

Fig. 1.

are necessary, but not sufficient, for the metric to be nonsingular at $r=r_{\mathrm{E}}$. Now

$$
\frac{\mathrm{d} c}{\mathrm{~d} r}=\frac{c}{V}\left(\frac{1}{2} \frac{\mathrm{d} V}{\mathrm{~d} r} \mp \frac{1}{\lambda}\right)
$$

where $\mp$ is for Schwarzschild or de Sitter, respectively.

Since $c \neq 0$ and $V=0$ at $r=r_{\mathrm{E}}$, we must have

$$
\frac{1}{\lambda}= \pm\left.\frac{1}{2} \frac{\mathrm{d} V}{\mathrm{~d} r}\right|_{r=r_{\mathrm{E}}},
$$

i.e. $\lambda=4 m$ for Schwarzschild, $\lambda=\sqrt{3 / \Lambda}$ for de Sitter. $\lambda$ is the «surface gravity" as defined in $\left({ }^{\top}\right)$, it is the acceleration of an inertial observer at $r_{E}$ relative to one at $r_{0}$.

Kruskal coordinates are well behaved at $r=r_{\mathrm{E}}$, but they are singular as $V \rightarrow 1\left(r=r_{0}\right)$, since $c \rightarrow 0$ there.

It can be shown that at $r=r_{\mathrm{E}}$ (and only at $r=r_{\mathrm{E}}$ ), $\partial / \partial t^{\prime}$ is a timelike Killing vector, and is tangent to geodesics (this point has been emphasized by Unruh $\left({ }^{8}\right)$ ). At $r=r_{0}$ (and only at $r=r_{0}$ ), $\partial / \partial t$ is a timelike Killing vector and tangent to a geodesic.

Since $t^{\prime}=\exp \left[r^{*} / \lambda\right] \cosh (t / \lambda)$ and $r^{\prime}=\exp \left[r^{*} / \lambda\right] \sinh (t / \lambda)$, we have the following algebra:

$$
\left[\frac{\partial}{\partial t^{\prime}}, \frac{\partial}{\partial t}\right]=\frac{1}{\lambda} \frac{\partial}{\partial r^{\prime}}
$$

(7) S. W. HawkIng: Commun. Math. Phys., 43, 199 (1975).

$\left(^{8}\right)$ W. G. UnRuh: Phys. Rev. D, 14, 870 (1976). 


$$
\begin{aligned}
& {\left[\frac{\partial}{\partial r^{\prime}}, \frac{\partial}{\partial t}\right]=\frac{1}{\lambda} \frac{\partial}{\partial t^{\prime}},} \\
& {\left[\frac{\partial}{\partial t^{\prime}}, \frac{\partial}{\partial r^{\prime}}\right]=0 .}
\end{aligned}
$$

This is the algebra of the two-dimensional Poincaré group. It holds everywhere, but only generates isometries at $r=r_{\mathrm{E}}$, when $V=0$. (At $r_{0}$, where $V=1$, $\partial / \partial t^{\prime} \rightarrow 0$ and $\partial / \partial r^{\prime} \rightarrow 0$, the algebra reduces to simple time translations, $\partial / \partial t$, which are Killing.)

Thus a freely falling observer at $r=r_{\mathrm{E}}, S^{\prime}$, would naturally define outgoing $s$ wave functions

$$
u_{\omega^{\prime}}^{\prime}=\frac{1}{2} \frac{1}{\sqrt{\pi \omega^{\prime}}} \exp \left[-i \omega^{\prime}\left(t^{\prime}-r^{\prime}\right)\right]
$$

(these functions only satisfy $\square u_{\omega^{\prime}}^{\prime}=0$ at $r=r_{\mathrm{E}}$ ). However a different freely falling observer, $S$, at $r=r_{0}$ would naturally define $s$-wave eigenfunctions by

$$
u_{\omega}=\frac{1}{2} \frac{1}{\sqrt{\pi \omega}} \frac{1}{r_{0}} \exp \left[-i \omega\left(t-r^{*}\right)\right]
$$

(these functions only satisfy $\square u_{\omega}=0$ at $r=r_{0}$ ). The $1 / r$ dependence of $u_{\omega}$ can be dropped without loosing any physics, since $S$ actually measures the expectation value of any $r$ independent operator, $\hat{A}$, as $\int_{S^{2}\left(r_{0}\right)} u_{\omega}^{*} \hat{A} u_{\omega} r_{0}^{2} \mathrm{~d} \Omega$. If a geodesic observer at $r^{*}=r_{0}^{*}(V=1)$ watches a plane wave $u_{\omega^{\prime}}^{\prime}$ coming from $r=r_{\mathrm{E}}$, he will find

$$
\alpha_{\omega \omega^{\prime}}=\left(\frac{\omega}{\omega^{\prime}}\right)^{1 / 2} \frac{\exp \left[-i \omega r_{0}^{*}\right]}{2 \pi} \int_{-\infty}^{\infty} \exp [i \omega t] \exp \left[-i \omega^{\prime}\left(t^{\prime}-r^{\prime}\right)\right] \mathrm{d} t
$$

where $t^{\prime}-r^{\prime}$ is a function of $t$ only. In $\left(t^{\prime}, r^{\prime}\right)$ coordinates, the world-line, $r_{0}=$ const, is given by

$$
t^{\prime}-r^{\prime}=-\exp \left[-\frac{1}{\lambda}\left(t-r_{0}^{*}\right)\right]
$$

where $r_{0}^{*}=r^{*}\left(r_{0}\right)=$ const.

Changing interpretation variables to $\tau=\exp \left[(1 / \lambda)\left(r_{0}^{*}-t\right)\right], 0<\tau<\infty$,

$$
\alpha_{\omega \omega^{\prime}}=\frac{\lambda}{2 \pi} \sqrt{\frac{\omega}{\omega^{\prime}}} \int_{0}^{\infty} \tau^{(-1-i \omega \lambda)} \exp \left[i \omega^{\prime} \tau\right] \mathrm{d} \tau
$$


If we give $\omega^{\prime}$ a small, positive, imaginary part, we can integrate by parts to get

$$
\alpha_{\left(\omega \omega^{\prime}\right.}=\frac{1}{2 \pi} \sqrt{\frac{\omega^{\prime}}{\omega}} \int_{0}^{x} \tau^{-i \omega \lambda} \exp \left[i \omega^{\prime} \tau\right] \mathrm{d} \tau,
$$

which is the integral arrived at in $\left(^{7}\right)$.

Thus

$$
\left|\alpha_{\omega \omega}\right|^{2}=\frac{1}{2 \pi} \frac{\lambda}{\omega^{\prime}} \frac{\exp [2 \pi \lambda \omega]}{\exp [2 \pi \lambda \omega]-1}
$$

$\beta_{\omega \omega^{\prime}}$ is obtained by analytically continuing $\alpha_{\omega \omega^{\prime}}$ to $\alpha_{-\omega, \omega^{\prime}}$ :

$$
\left|\beta_{\left(\omega v^{\prime}\right.}\right|^{2}=\frac{1}{2 \pi} \frac{\lambda}{\omega^{\prime}} \frac{1}{1-\exp [2 \pi \lambda \omega]} .
$$

This is the thermal radiation formula obtained by Hawking $\left({ }^{7}\right)$ with $k_{\mathrm{B}} T=1 / 2 \pi \hbar \lambda$ $\left(k_{\mathrm{B}}=\right.$ Boltzmann's constant $)$.

In the original work of Hawking on the Schwarzschild metric, time asymmetry was imposed by assuming that the black hole formed under gravitational collapse. Here time asymmetry is imposed since the observer at $r_{0}$ only looks at outcoming modes. For the de Sitter metric, the same result holds, provided the observer only looks at $u^{\prime}$ modes coming from the past.

From the point of view of group theory, the Fourier integral performed to calculate the coefficients, $\beta_{\text {wo' }}$, is seen as a unitary transformation between different representations of the two-dimensional Poincaré group. Seen in this light, the phenomenon of particle creation can be deduced from a knowledge of the algebra only, since this is sufficient for the construction of the unitary representations (of course the algebra itself is only constructed from a knowledge of the global structure of the metric). The fact that the positive energy eigenstates of $\partial / \partial t^{\prime}$ contain negative-energy eigenstates of $\partial / \partial t$ becomes a statement of group representation theory.

\section{3. - New applications.}

The previous presentation has been made general enough for a treatment of other metrics of a similar form. For example, the Bertotti-Robinson metric $\left(^{9,10}\right)$

$$
\mathrm{d} s^{2}=-\left(1-\alpha^{2} z^{2}\right) \mathrm{d} t^{2}+\left(1-\alpha^{2} z^{2}\right)^{-1} \mathrm{~d} z^{2}+\left(1+\alpha^{2} z^{2}\right) \mathrm{d} x^{2}+\left(1+\alpha^{2} z^{2}\right)^{-1} \mathrm{~d} y^{2}
$$

(') B. Bertotti: Phys. Rev., 116, 1331 (1959).

${ }^{\left({ }^{10}\right)}$ I. Robinson: Bull. Acad. Polon. Sci., Ser. Math. Astr. Phys., 7, 351 (1959). 
has the same essential features with $V(z)=\left(1-\alpha^{2} z^{2}\right)$. This is the metric 'for a static, homogeneous, electromagnetic field, with

$$
\boldsymbol{E}^{2}+\boldsymbol{B}^{2}=2 \alpha^{2}
$$

If $x$ and $y$ are suppressed, it is just the two-dimensional de Sitter metric. There will be an event horizon at $z=1 / \alpha$ and thermal particle production with

$$
k_{\mathrm{B}} T=\frac{\alpha}{2 \pi \hbar}
$$

However, if the electromagnetic field were to become strong enough for appreciable temperatures to develop, it has to be so strong that its energy density is much higher than $2 m_{\mathrm{e}} / \lambda_{\mathrm{e}}^{3}$ where $m_{\mathrm{e}}$ is the mass of the electron and $\lambda_{\mathrm{e}}=\hbar / m_{\mathrm{e}} c$ the electron Compton wavelength. Such strong fields would spontaneously create $\mathrm{e}^{+} \mathrm{e}^{-}$pairs, which would nullify the field, i.e. the vacuum could not sustain such strong fields.

Other metrics which would display the same phenomenon are the "generalized" Reissner-Nortstrom-de Sitter metrics $\left({ }^{11}\right)$ :

$$
\mathrm{d} s^{2}=-V(r) \mathrm{d} t^{2}+V(r)^{-1} \mathrm{~d} r^{2}+\left(\mathrm{d} x^{2}+\Sigma^{2}(\chi, \varepsilon) \mathrm{d} \varphi^{2}\right)
$$

where

$$
\Sigma(\chi, \varepsilon)=\frac{1}{\sqrt{\varepsilon}} \sinh (\sqrt{\varepsilon} \chi)
$$

and

$$
V(r)=\left(-\varepsilon-\frac{2 m}{r}+\frac{e^{2}}{r^{2}}-\frac{\Lambda}{3} r^{2}\right) \quad \varepsilon= \pm 1,0
$$

However such metrics are difficult to interpret physically. Other metrics are the Kerr metric, discussed in $\left(^{7}\right)$ and the Taub-NUT metric $\left(^{3}\right)$. For the latter, however, the region in which the radiation would be observed has closed timelike curves, and so the physical interpretation is obscure.

\section{4. - Conclusion.}

In conclusion, it has been argued that the phenomenon of Hawking radiation can be understood from the point of view of the representation theory of the two-

(11) D. Kramer, H. Stephani, M. MacCallum and Herlt: Exact Solutions of Einstein's Field Equations (DVB, 1980). 
dimensional Poincaré group. This necessitates the analysis of two inequivalent geodesic world-lines. To perform the unitary transformations between the representations it is necessary that the tangent to one observer's geodesic be Killing along the whole geodesic, though the other world-line only has a Killing tangent vector at the event horizon.

The Killing symmetry appears essential for an understanding of the problem. It is not necessary however that both observers be geodesic, as witnessed by the thermal radiation seen by a uniformly accelerating observer in Minkowski space $\left.{ }^{(}\right)$, where the role of the Poincare group is manifest.

Finally, let us emphasize that the generators of the two-dimensional Poincaré group are defined globally, however, they only generate isometries at the event horizon $r=r_{\mathrm{E}}$ and at $r=r_{0}$.

$$
* * *
$$

The author would like to thank I. M. Benn for comments which led to this investigation.

\section{- RIASSUNTO (*)}

Si mostra che, per spazi-tempo statici, si può comprendere il fenomeno della radiazione di Hawking nei termini delle rappresentazioni del gruppo di Poincaré a due dimensioni. Il vettore di base ad energia zero di una rappresentazione contiene vettori di base ad energia positiva di un'altra rappresentazione. Un'azione del gruppo di Poincaré a due dimensioni è globalmente definita su tutto lo spazio-tempo, ma genera solo isometrie all'orizzonte degli eventi e nella regione asintoticamente piatta (dove degenera in semplici translazioni temporali). Si discutono alcuni semplici spazi-tempo.

(*) Traduzione a cura della Redazione.

\section{Теоретико-групповой подход к проблеме излучения черной дыры.}

Резюме (*). - Показывается, что для статических пространства-времени явление излучения Хокинга можно понять в терминах представлений двумерной группы Пуанкаре. Базисный вектор нулевой энергии одного представления содержит базисные векторы с положительной энергией другого представления. Действие двумерной группы Пуанкаре глобально определяется на всем пространстве-времени, но изометрии образуются только на горизонте событий и в асимптотически плоской области (где действие производит“ простые временные трансляции). Обсуждаются некоторые простые типы пространства-времени.

(*) Переведено редакцией. 